\title{
Use of acetazolamide to decrease cerebrospinal fluid production in chronically ventilated patients with ventriculopleural shunts
}

\author{
E Carrion, J H Hertzog, M D Medlock, G J Hauser, H J Dalton
}

\begin{abstract}
Acetazolamide (ACTZ), a carbonic anhydrase inhibitor, has been shown to decrease cerebrospinal fluid (CSF) production in both in vivo and in vitro animal models. We report two children with hydrocephalus who experienced multiple shunt failures, and who had externalised ventriculostomy drains (EVD) prior to ventriculopleural shunt placement. The effects of increasing doses of ACTZ on CSF production and subsequent tolerance to ventriculopleural shunts were evaluated. The patients had a $48 \%$ and a $39 \%$ decrease in their EVD CSF output when compared to baseline with maximum ACTZ dose of $75 \mathrm{mg} / \mathrm{kg} / \mathrm{day}$ and $50 \mathrm{mg} / \mathrm{kg} /$ day, respectively $(p<0.05)$. This is the first report of change in CSF volume in children after extended treatment with ACTZ. ACTZ treatment in mechanically ventilated paediatric patients with hydrocephalus may improve tolerance of ventriculopleural shunts and minimise respiratory compromise. Potassium and bicarbonate supplements are required to correct metabolic disturbances.

(Arch Dis Child 2001;84:68-71)
\end{abstract}

Keywords: acetazolamide; cerebrospinal fluid; hydrocephalus; ventriculopleural shunts

Department of

Pediatrics, Division of Pediatric Critical Care and Pulmonary

Medicine, Georgetown

University Medical

Center, Washington,

DC, USA

E Carrion

J H Hertzog

G J Hauser

H J Dalton

Department of Neurosurgery, Georgetown University Medical Center M D Medlock

Correspondence to: Dr E Carrion, Children's National Medical Center, Department of Critical Care Medicine, 111 Michigan Avenue, NW Washington, DC 20010-2970, USA kikecarrion@pol.net

Accepted 13 July 2000
Congenital and acquired obstructive hydrocephalus are common problems in children, with an incidence of $0.4-0.8$ per 1000 liveborns and stillbirths. ${ }^{1}$ The majority of these patients are managed with ventriculoperitoneal shunts. The ability of the peritoneal membranes to absorb cerebrospinal fluid (CSF) may be decreased after intra-abdominal infections or surgery, such that a ventriculoperitoneal shunt may not provide adequate decompression of the ventricles. In this situation, CSF may need to be drained from the ventricle into other body cavities, such as the pleural space. The success of ventriculopleural shunts may be limited by the rate of absorption of CSF by the pleural membranes. Respiratory insufficiency may develop with the accumulation of large volumes of CSF in the pleural space. Therefore, attempts to limit CSF production may be beneficial in this situation.

Acetazolamide (ACTZ), a carbonic anhydrase inhibitor, has been shown to decrease CSF production in animal models and is used in both adults and children to treat conditions such as pseudotumor cerebri. ${ }^{2}{ }^{3}$ There are, however, no studies reporting the change in CSF volume produced by children after extended treatment with ACTZ.

We report our use of ACTZ in two children who had experienced multiple ventriculoperitoneal shunt failures and who were temporarily managed with externalised ventriculostomy drains (EVD) prior to ventriculopleural shunt placement. We evaluated the effect of ACTZ on CSF production and the subsequent tolerance of the ventriculopleural shunt.

\section{Case reports}

Patient 1 was a 1 year old male born at 24 weeks gestation with multiple medical problems, including bronchopulmonary dysplasia (BPD) and respiratory failure requiring mechanical ventilatory support via a tracheostomy. He also had a right femoral Broviac type indwelling central catheter for parenteral nutrition and medications. He developed hydrocephalus secondary to an intraventricular haemorrhage. A ventriculoperitoneal shunting was complicated by several episodes of obstruction and infection. In addition, he developed necrotising enterocolitis, which further scarred the peritoneum. As an alternative to a ventriculoperitoneal shunt, a ventriculoatrial shunt was placed. However, a venous thrombus developed, resulting in superior vena cava syndrome, necessitating removal of the shunt and placement of an EVD.

As placement of the shunt into the peritoneum or vascular spaces was no longer a viable option, we anticipated that a ventriculopleural shunt would be necessary. Given the child's respiratory insufficiency, there was concern that a large collection of CSF in the pleural space would not be tolerated. Therefore, he was treated promptly with ACTZ in an attempt to decrease CSF production. We used an initial dose of $30 \mathrm{mg} / \mathrm{kg} /$ day for four days, subsequently increased to $50 \mathrm{mg} / \mathrm{kg} /$ day for 13 days and then to $75 \mathrm{mg} / \mathrm{kg} /$ day. CSF volume measurements during ACTZ treatment were available for 35 days. Table 1 and fig 1 show the effect of ACTZ on CSF volume. Baseline measurements used for comparison reflect CSF volumes measured during previous EVD placements. When compared to baseline, there was a $48 \%$ decrease in EVD output at the maximal dose of ACTZ ( $p=0.036$ by Student's $t$ test). Serum electrolytes were checked daily. Enteral potassium (up to $4 \mathrm{mmol} / \mathrm{kg} /$ day) and bicarbonate (up to $8.4 \mathrm{mmol} / \mathrm{kg} /$ day) were given to maintain normal serum electrolyte concentrations. There were no significant 
Table 1 Effect of increasing ACTZ dose on EVD output per hour for each patient

\begin{tabular}{lll}
\hline & \multicolumn{2}{l}{ EVD output/h $(\mathrm{ml})$} \\
\cline { 2 - 3 } Acetazolamide (mg/kg/day) & Patient 1 & Patient 2 \\
\hline 0 & $8.0(0.0)$ & $9.1(0.2)$ \\
30 & $4.6(0.2)(-42 \%)$ & $6.08(0.4)(-16 \%)$ \\
50 & $5.6(0.7)(-30 \%)$ & $5.73(0.5)^{\star}(-39 \%)$ \\
\hline 5 & $4.2(0.2)^{\star}(-48 \%)$ & N/A \\
\hline
\end{tabular}

SE values in brackets.

${ }^{\star} \mathrm{p}<0.05$ versus baseline.

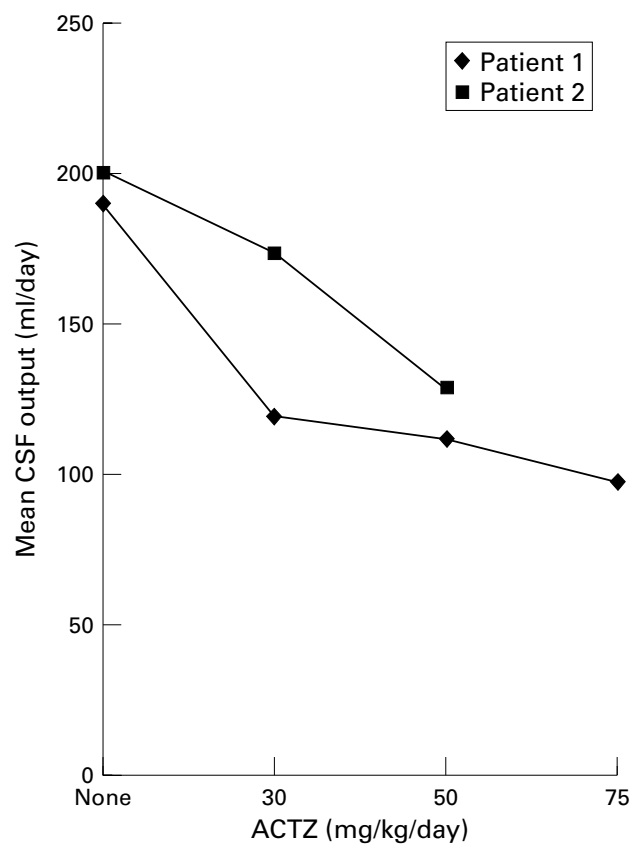

Figure 1 Effect of increasing ACTZ dose on daily EVD output.

complications from ACTZ therapy. After placement of the ventriculopleural shunt, no appreciable accumulation of pleural fluid was noted on chest radiograph. The patient's respiratory status remained stable. Head growth and ventricular size were kept within stable limits during the observation period. The child suffered no further episodes of shunt failure. Three months after placement of the ventriculopleural shunt, he developed bacterial pneumonia and died from respiratory failure. There was no evidence of a pleural effusion.

Patient 2 was a 1 year old female with myelomeningocele and Arnold-Chiari malformation type II. She had vocal cord dysfunction and abnormal central respiratory drive necessitating mechanical ventilatory support via a tracheostomy; and a left subclavian Broviac type indwelling central catheter for antibiotics and parenteral nutrition. A ventriculoperitoneal shunt was placed on the first day of life for management of her obstructive hydrocephalus. She subsequently experienced multiple episodes of shunt obstruction and shunt infection. In addition, she developed an abdominal wound infection and peritonitis in association with a jejunostomy tube placement. Due to the failure of the peritoneum to absorb CSF adequately, a ventriculoatrial shunt was placed. However, she experienced bacteraemia and infection of this shunt as well, necessitating removal and placement of an EVD.
Given these failures, a ventriculopleural shunt was considered. Her third ventricle was too small to consider a third ventriculostomy. We were concerned at a history of CSF output (>200 ml/day) from prior EVD placement and the impact that this volume of pleural fluid might have on her respiratory status. Therefore, in an attempt to diminish CSF production, ACTZ at a dose of $30 \mathrm{mg} / \mathrm{kg} /$ day was prescribed with EVD placement. After 13 days, the dose of ACTZ was increased to $50 \mathrm{mg} / \mathrm{kg} /$ day. Placement of a ventriculopleural shunt was performed five days later. Figure 1 and table 1 show the impact of ACTZ treatment on the volume of CSF drained from the EVD. Compared to baseline, there was a $39 \%$ decrease in CSF volume with $50 \mathrm{mg} / \mathrm{kg} /$ day of ACTZ ( $\mathrm{p}=0.001$ by Student's $t$ test). Enteral potassium (up to $5.3 \mathrm{mmol} / \mathrm{kg} /$ day) and bicarbonate (up to $8 \mathrm{mmol} / \mathrm{kg} /$ day) were given to maintain normal serum electrolyte concentrations which were monitored daily. There were no significant complications from ACTZ therapy. Within two days of ventriculopleural shunt placement, we noted a significant pleural effusion on chest $x$ ray, with a corresponding deterioration in her respiratory status. Treatment included an increase in supplemental oxygen and positive end expiratory pressure. There was a gradual decrease in the size of the pleural effusion over the next several days. The positive end expiratory pressure was returned to baseline without reaccumulation of the pleural effusion. The child was subsequently discharged home with mechanical ventilatory support and ACTZ. Over the past two years, her head circumference and ventricular size have been within stable limits and she continues on acetazolamide $(50 \mathrm{mg} / \mathrm{kg} /$ day $)$. She has been admitted to the hospital three times with large pleural effusions associated with intercurrent respiratory syncitial virus pneumonia. On one occasion the effusion resolved spontaneously and on the two others a single thoracentesis was sufficient with no recurrence of the pleural effusions.

\section{Discussion}

The routine surgical management of hydrocephalus includes the placement of a shunting device to bypass the obstruction and connect the ventricles to a body cavity where CSF can be absorbed. Most commonly the peritoneal space is used because of the large peritoneal surface area available for absorption. ${ }^{4}$ Peritonitis and intra-abdominal surgery may significantly decrease the absorptive capability of the peritoneum, resulting in the failure of subsequently placed ventriculoperitoneal shunts. In this situation, other body cavities must be employed.

Ventriculopleural shunting was first described by Heile in $1914 .{ }^{5}$ Since then, this alternative approach had been used with success in a selected population of patients. The most common complication described with this shunt is the development of a unilateral pleural effusion. ${ }^{67}$ It has been proposed that this procedure be limited to older patients, because younger patients may be unable to 
absorb significant CSF volumes from the pleural space. ${ }^{5}$ Certainly large pleural effusions will compromise respiratory function, especially in infants who have less respiratory reserve than adults. Patients with chronic respiratory failure receiving mechanical ventilatory support may be even less tolerant of fluid accumulations in the pleural space. Thus, for this particular population, ventriculopleural shunts are not ideal. In some patients, however, there may be no other option than to use the pleural space. In this situation, attempting to decrease pleural fluid volumes by decreasing CSF production may improve tolerance of a ventriculopleural shunt.

ACTZ has been used for years to limit CSF production and it has been studied extensively both in vivo and in vitro. It acts to decrease CSF production by altering blood flow to the choroid plexus. Depending on the experimental model, CSF production decreases by $40-90 \% .^{8-10}$ ACTZ has a rapid onset of action. In dogs, $10 \mathrm{mg} / \mathrm{kg}$ of ACTZ produces a significant decrease in the production and absorption of CSF within three hours of ingestion. ${ }^{10}$

Our report is unique in that we know of no other study that evaluated changes in CSF volume over time with the extended use of ACTZ in human infants. Bucholz and Pittman ${ }^{11}$ briefly discuss the use of ACTZ to decrease CSF output in a 29 month old hydrocephalic infant with ventriculoperitoneal shunt and ascites. They externalised the shunt and subsequently administered ACTZ (dose used not reported) on postoperative days 5 to 7 . CSF volume was reduced by $25 \%$ during this brief period. As ACTZ therapy resulted in hypokalaemia and hyperchloraemia, the drug was discontinued. Our two cases differ in that CSF volumes were measured during treatment with ACTZ for 35 and 18 days, respectively. Other studies have evaluated the effect of ACTZ on parameters other than direct volume measurement. In a study performed by Shinnar et al, infants with hydrocephalus received ACTZ $(100 \mathrm{mg} / \mathrm{kg} / \mathrm{day})$ and frusemide (1 mg/kg/ day). ${ }^{12}$ This avoided ventricular shunting in more than $50 \%$ of the cases. We emphasise that the use of medical treatment to avoid surgery may be hazardous, and deciding to adopt this modality of therapy needs to be balanced against the seriousness of the clinical circumstances. In a recent international randomised controlled trial, the combined use of acetazolamide and frusemide in preterm infants with posthaemorrhagic ventricular dilatation in order to avoid surgical management was associated with a higher rate of shunt placement and increased neurological morbidity. ${ }^{13}$ Based on our limited experience, we consider it reasonable to consider ACTZ treatment when there is a need to decrease CSF volume in infants and children.

Another important aspect of our report is that ACTZ seemed to improve tolerance of ventriculopleural shunts in infants with chronic lung disease requiring mechanical ventilatory support. To our knowledge, this is the first encouraging report regarding the use of ventriculopleural shunts in infants, especially those with an increased risk of morbidity as a result of their pulmonary disease. Previous reports of ventriculopleural shunts in children describe pleural effusions as a common complication and recommend limiting its use to older patients. ${ }^{71415}$ While we did not study the effect of ACTZ treatment on pleural effusion volume after ventriculopleural shunt placement, we speculate that ACTZ was important for the successful use of ventriculopleural shunts in our two patients. In our study, ACTZ apparently diminished CSF production significantly enough to avoid apparent respiratory compromise in patient 1 and to improve respiratory function in patient 2. Therefore, ACTZ should be considered when a ventriculopleural shunt is anticipated in small children, especially when the child has concurrent respiratory difficulties.

An important side effect of ACTZ is the development of hyperchloraemic metabolic acidosis with hypokalaemia. ${ }^{16}$ Therefore, it is important to closely monitor serum electrolytes during ACTZ therapy and to provide potassium and bicarbonate replacement as needed to maintain normal electrolyte balance. In both of our cases, we were able to maintain normal electrolyte values with this strategy. In addition, the diuretic effect of ACTZ must be kept in mind, with careful attention given to the patient's overall fluid balance. Intake and output measurements while receiving ACTZ at different doses were compared for each patient (data not shown). Statistical analysis revealed no significant difference between each group ( $p>0.05$ by Student's $t$ test).

In summary, the use of ACTZ resulted in a significant decrease in the CSF volume measured from EVDs in our patients. This reduction minimised the symptoms of respiratory compromise commonly associated with the use of ventriculopleural shunts in infants. These findings suggest that ACTZ can be used safely in mechanically ventilated paediatric patients with hydrocephalus and ventriculopleural shunts to decrease CSF accumulation in the pleural space. This therapy may improve tolerance to ventriculopleural shunts. Potassium and bicarbonate supplements are required to correct acid-base disturbances. With close monitoring, ACTZ is a safe therapy for the management of pleural effusions associated with ventriculopleural shunts.

1 Schrander-Stumpel C, Fryns JP. Congenital hydrocephalus: osology and guidelines for clinical approach and genetic counseling. Eur f Pediatr 1998;157:355-62.

2 Sorensen PS, Krogsaa B, Gjerris F. Clinical course and prognosis of pseudotumor cerebri. A prospective study of 24 patients. Acta Neurol Scand 1988;77:164-72.

3 Schoeman JF. Childhood pseudotumor cerebri: clinical and intracranial pressure response to acetazolamide and furosemide treatment in a case series. $\mathcal{F}$ Child Neurol 1994; 9:130-4.

4 Fandino J, Botana C, Viladrich A, et al. VPS in the treatment of hydrocephalus in children. Results and complications. An Esp Pediatr 1990;32:325-8.

5 Sanders DY, Summers R, DeRouen L. Symptomatic pleural collection of CSF caused by a VPL shunt. South Med $\mathcal{F}$
1997;90:345-6.

6 Willison CD, Kopitnik TA, Gustafson R, et al. Ventriculopleural shunting used as a temporary diversion. Acta Neurochir 1992;115:67-8. 
7 Piatt JH Jr. How effective are ventriculopleural shunts? Pediatr Neurosurg 1994;21:66-70.

(1) bain bain on CSF production rate in newborn dogs. Am $\mathcal{F}$ Physiol 1972;223:503-6.

9 Knuckey NW, Fowler AG, Johanson CE, et al. Cisterna magna microdialysis of $22 \mathrm{Na}$ to evaluate ion transport and CSF hemodynamics. F Neurosurg 1991;74:965-71.

10 Watanabe K, Ishikawa S, Kimoto T, et al. Production and absorption rate of CSF in spinal subarachnoid space of dog. No Shinkeigeka 1976;4:351-8.

11 Bucholz R, Pittman T. Endoscopic coagulation of choroid plexus using Nd:YAG laser: initial experience and proposal for management. Neurosurgery 1991;28:421-6.
12 Shinnar S, Gammon K, Bergman EW, et al. Management of hydrocephalus in infancy: use of acetazolamide and furosem-

3 International PHVD Drug Trial Group. International randomized controlled trial of acetazolamide and furosemide in posthaemorrhagic ventricular dilatation in infancy. Lancet 1998;352:433-40.

14 Venes JL, Shaw RK. Ventriculopleural shunting in management of hydrocephalus. Childs Brain 1979;5:45-50.

15 Hoffman HJ, Hendrick EB, Humphreys RP. Experience with ventriculopleural shunts. Childs Brain 1983;10:404-13.

16 Futagi Y, Otani K, Abe J. Growth suppression in children receiving acetazolamide with antiepileptic drug. Pediatr Neurol 1996;15:323-32.

\section{Rapid responses}

Letters on the following papers have been published recently as rapid responses on the $A D C$ website. To read these letters visit www.archdischild.com and click on "Read rapid responses":

An association between sudden infant death syndrome (SIDS) and Helicobacter pylori infection. J R Kerr, A Al-Khattaf, A J Barson, et al. Arch Dis Child 2000;83:429-34.

The role of lumbar puncture in meningococcal disease. W I C Sam. Arch Dis Child 2000;83:369.

Scrotal temperature is increased in disposable plastic lined nappies. C-J Partsch, $M$ Aukamp, W G Sippell. Arch Dis Child 2000;83:364-8.

Respiratory syncytial virus infection in high risk infants and the potential impact of prophylaxis in a United Kingdom cohort. S J Clark, M W Beresford, N V Subhedar, et al. Arch Dis Child 2000;83:313-16.

Quality of life in spina bifida: importance of parental hope. H M Kirpalani, P C Parkin, A R Willan, et al. Arch Dis Child 2000;83:293-7.

The physical punishment of children. D Elliman, M A Lynch. Arch Dis Child 2000;83:196-8.

Effect of oral glucocorticoid treatment on serum inflammatory markers in acute asthma. A Sahid El-Radhi, C L Hogg, Jatinder K Bungre, et al. Arch Dis Child 2000;83:15862.

Hospitalisation for RSV infection in ex-preterm infants: implications for use of RSV immune globulin. M Thomas, A Bedford-Russell, M Sharland. Arch Dis Child 2000;83:1227.

Dietary products used in infants for treatment and prevention of food allergy. J Salazar de Sousa, B Koletzko, A Host. Arch Dis Child 2000;83:87.

If you would like to post an electronic response to these or any other articles published in the journal, please go to the website, access the article in which you are interested, and click on "eLetters: Submit a reponse to this article" in the box in the top right hand corner. 